\title{
Percepción general de la seguridad vial en la ciudad de Loja (Ecuador)
}

\author{
Perception of road safety in Loja city (Ecuador).
}

Belizario Zárate

Universidad Técnica Particular de Loja

(Loja - Ecuador)

Julio González

Universidad Técnica Particular de Loja

(Loja - Ecuador)

\author{
Yasmany García-Ramírez \\ Universidad Técnica Particular de Loja \\ (Loja - Ecuador) \\ ydgarcia1@utpl.edu.ec
}

Soledad Segarra

Universidad Técnica Particular de Loja

(Loja - Ecuador)

Revista Cumbres Vol.4 №1

Versión impresa ISSN 1390-9541

Versión electrónica ISSN 1390-3365

http://investigacion.utmachala.edu.ec/revistas/index.php/Cumbres 


\section{RESUMEN}

El gobierno ecuatoriano ha llevado a cabo varias acciones para prevenir y reducir el número de muertes por accidentes de tránsito. Esas acciones se han realizado sin considerar la percepción que tienen los usuarios viales frente a la seguridad vial en general. Al conocerse, los recursos podrían administrarse de mejor manera. En consecuencia, esta investigación explora la percepción general que tienen los usuarios viales en una ciudad del Ecuador, para ello, se aplicaron encuestas a 1197 personas (peatones y conductores) en varios sectores de la Ciudad de Loja. En esa encuesta se incluyeron preguntas relacionadas con las sensaciones, emociones y comportamientos de los usuarios en la vía, y su opinión con respecto a los accidentes de tránsito y a las leyes de tránsito vigentes. Los resultados muestran que a la mayoría de conductores les disgusta la forma de conducir de otros conductores, sin embargo, justifican sus maniobras arriesgadas. En cuanto a sensaciones, la mayoría de peatones sienten miedo cuando circulan por la calle, mientras que los acompañantes o copilotos sienten miedo y estrés. Por otro lado, la mayoría de la muestra analizada no está de acuerdo con las leyes de tránsito en general, especialmente con la de consumo de bebidas alcohólicas y del exceso de velocidad. Asimismo, se detectaron dos grupos de mayor riesgo: menores a 30 años y los mayores a 55 años, en los cuales debiera enfocarse las campañas de seguridad vial.

Palabras clave: percepción de la seguridad vial, Loja (Ecuador), accidentes de tránsito.

\section{ABSTRACT}

The Ecuadorian government has carried out several actions to prevent and reduce the number of deaths due to traffic accidents. Such actions have been conducted without considering the perception road users have about road safety. If stakeholders learn about their opinion, resources can be better administrated. In consequence, this research explores the overall road users' perception in a city in Ecuador. For this purpose, surveys were applied to 1197 people (pedestrians and drivers) in different sectors of Loja city. The survey included questions related to the feelings, emotions, and behaviors of road users and their opinion regarding traffic accidents and current traffic laws. The results show that most drivers dislike the way other drivers drive, however, they justify their risky maneuvers. In terms of feelings, most pedestrians indicated that they are afraid when they walk down the streets, while drivers' companions or co-pilots feel fear and stress. The majority of the participants does not agree with the current traffic laws, especially with the alcoholic beverages and speeding regulations. Finally, two groups with the highest road unsafety risk were detected. They are people who are under 30 years of age and those over 55; being these groups the target for road 
safety campaigns.

Keywords: Road safety perception, Loja (Ecuador), traffic accidents.

\section{INTRODUCCIÓN}

La seguridad vial pretende prevenir los accidentes de tránsito mediante acciones y/o mecanismos que permitan una interacción armónica entre el conductor, peatón, ciclista, vehículo, tránsito y demás elementos del entorno vial. La falta de esa interacción armónica podría llevar a un aumento en la frecuencia de accidentes de tránsito. Es así, que un informe de la Organización Mundial de la Salud (OMS, 2015) revela que alrededor de 1,3 millones de personas fallecen anualmente a consecuencia de un accidente de tránsito en el mundo. En ese informe, en Ecuador existieron 3.072 accidentes mortales reportados en el 2013, lo que representó 20,1 muertes por cada 100.000 habitantes, lo cual ubicó al país en el cuarto puesto con mayor número de accidentes a nivel de América del Sur. Con el fin de estabilizar y luego reducir el número de muertos por accidentes de tránsito en todo el mundo, la Organización de las Naciones Unidas proclamó el 2011-2020 como el Decenio de Acción para la Seguridad Vial (ONU, 2011). En este acuerdo se propusieron 5 pilares fundamentales: gestión de la seguridad vial, vías de tránsito y movilidad más seguras, vehículos más seguros, usuarios de vías de tránsito más seguros y respuesta tras los accidentes.

En relación a este acuerdo, varios países realizaron y están realizando acciones para cumplir con esos objetivos, no obstante, estas acciones deben estar acorde a la realidad de cada país, por lo que no pueden ser las mismas (Wegman, 2017). En Ecuador, en todas las provincias del país se aplicaron varias acciones en seguridad vial: campañas de información ("Señalética Vial", "Mira al frente", "Párale el carro"), aumento en el precio de las bebidas alcohólicas (Ley $\mathrm{N}^{\circ} 744,2016$ ), endurecimiento de las penas y multas por infracciones de tránsito (Código Orgánico Integral Penal, 2014) y mayor control policial, lo que ha llevado a una reducción del número de accidentes fatales a nivel nacional (ANT, 2016a).

El gobierno ecuatoriano, al igual que varios países de América Latina, aplicaron estas acciones basándose principalmente en las estadísticas de accidentes de tránsito, lo cual no necesariamente conduce a la reducción de accidentes de tránsito. Antes, durante y después de la aplicación de estas acciones, los gobiernos debieran conocer la opinión de los usuarios para realizar modificaciones de manera oportuna. Esta opinión se refiere a la percepción que tiene la población acerca de las acciones tomadas por el gobierno y de la seguridad vial, la cual no es homogénea, dado que puede ser afectada por cuestiones culturales, falta de conocimiento, diferencia generacional, entre otras.

En cada ciudad del país, la división provincial del Ministerio de Transporte y Obras Públicas y los gobiernos locales, se encargaron de aplicar o hacer cumplir las acciones nacionales, y de realizar campañas de seguridad vial específicas para cada ciudad. En estos gobiernos locales, de manera similar 
que el gobierno nacional, se conoce muy poco acerca de la opinión de sus usuarios viales, principalmente por sus limitaciones financieras y en algunos casos no se dispone de personal calificado.

Entonces, dada la importancia de la opinión de los usuarios en seguridad vial, esta investigación explora la percepción que tienen los usuarios viales en una ciudad del Ecuador. El estudio fue realizado con una muestra de la Ciudad de Loja, la cual es la quinta provincia con mayor densidad poblacional (41 hab/km2) del país. Aunque existen relaciones que se pueden ser comunes con otras ciudades, este estudio sólo debe ser usado como referencia, por lo que se sugiere realizar su propio estudio utilizando esta metodología

\section{MATERIALES Y MÉTODOS}

\section{Sitio del estudio}

La Ciudad de Loja está ubicada en la Provincia de Loja, la cual tiene 11.062,73 km2 de extensión y una población de 448.966 habitantes. Tiene una densidad poblacional de $41 \mathrm{hab} / \mathrm{km} 2$ y una densidad vehicular de 9260 vehículos por cada 100.000 habitantes y una frecuencia de accidentalidad de 125 accidentes por cada 100.000 habitantes (INEC, 2010). Se eligió esta ciudad debido a que la densidad poblacional está cerca de la densidad promedio del país y de la mayoría de poblaciones en América del Sur, Centroamérica y el Caribe (FAO, 2015).

\section{Instrumento}

Para la recolección de datos se utilizó una encuesta dirigida a peatones y conductores de la Ciudad de Loja, la cual fue respondida durante varios meses del 2015. Las preguntas de la encuesta fueron elaboradas en base a un estudio realizado en Colombia denominado: Primer estudio de comportamiento y seguridad vial (IPSOS, 2010). La encuesta tiene preguntas relacionadas con las sensaciones, emociones y comportamientos de los usuarios en la vía; y su opinión con respecto a los accidentes de tránsito y a las leyes de tránsito vigentes, y con la seguridad vial en general.

\section{Tamaño muestral}

Para estimar el tamaño muestral se utilizó la siguiente ecuación 1, donde N: población del cantón, o: desviación estándar, z: nivel de confianza, y e: error muestral.

$$
n=\frac{N \sigma^{2} Z^{2}}{(N-1) e^{2}+\sigma^{2} Z^{2}} \quad \text { Ecuación 1 }
$$

El cantón Loja tiene 214.855 habitantes (INEC, 2010) y con una desviación estándar de 0,5, con un nivel de confianza de 2,575 (99\% de confiabilidad) y un error del $4 \%$ se obtiene un tamaño muestral de 1.031 encuestas, sin embargo, se recolectaron 1.197 encuestas considerando que algunas encuestas podían ser descartadas por errores u omisiones del entrevistador. 


\section{Recolección de datos}

Treinta y tres entrevistadores realizaron las encuestas desde febrero a noviembre del 2015, los cuales fueron distribuidos en diversos sectores de la ciudad. El 52\% de los encuestados fueron mujeres y el 48\% fueron hombres, los cuales están cercanos a distribución poblacional por sexo de la Provincia de Loja y del país. Principalmente, los entrevistados fueron amas de casa, artesanos, estudiantes, ingenieros, empresarios, licenciados, relacionados con la educación y relacionados con la salud.

La edad de los entrevistados estuvo entre 18 a 67 años, los cuales se dividieron de 5 en 5 a partir de los 17,5 años. En el eje horizontal de las figuras o en la descripción de las tablas se muestra el valor promedio de esas clases. Cuando no hubo ninguna diferencia significativa entre grupos de edades, se analizó el promedio de las respuestas de todas las edades. De los 1.197 encuestados, 526 fueron conductores regulares y el resto fueron peatones.

\section{RESULTADOS Y DISCUSIÓN}

\section{Sensaciones o comportamientos de los conductores en la vía}

Las sensaciones y/o comportamientos de los conductores también son importantes en la vía, dado que pueden ocasionar accidentes de tránsito. Es así que, se les preguntaron a 526 conductores acerca de estos dos temas en varias ocasiones. Dada la homogeneidad de las respuestas entre conductores de diferentes edades, se decidió analizar sus respuestas como un solo grupo (Ver Tabla 1).

Tabla 1: Sensaciones o comportamiento mostrados por los conductores encuestados cuando utilizan las

\begin{tabular}{|l|c|c|c|c|}
\hline \multirow{2}{*}{ Sensación o comportamiento } & \multicolumn{4}{c|}{ Porcentaje de encuestados (\%) } \\
\cline { 2 - 5 } & Siempre & $\begin{array}{c}\text { Algunas } \\
\text { veces }\end{array}$ & $\begin{array}{c}\text { Casi } \\
\text { nunca }\end{array}$ & Nunca \\
\hline No hay nada mejor que una calle sin tráfico & 26 & 35 & 22 & 17 \\
\hline Manejar lo tomo como un placer y no como algo aburrido & 18 & 25 & 29 & 28 \\
\hline Me gusta que me vean manejando & 8 & 23 & 31 & 38 \\
\hline Me gusta competir con el resto & 3 & 11 & 27 & 59 \\
\hline
\end{tabular}

En la Tabla 1, como era de esperarse, la mayoría de conductores (siempre + algunas veces) disfrutan de una calle sin tráfico, lo cual puede ser difícil de encontrarse, sin embargo, en condiciones normales de tránsito, la mayoría de personas no disfrutan de la conducción (casi nunca + nunca), posiblemente al carga mental asociada a las tareas de conducción. Por otro lado, un $31 \%$ (siempre + algunas veces) les gusta que los vean manejando, lo cual suele asociarse con un estatus social alto y el 14\% (siempre + algunas veces) le gusta competir con el resto, lo cual es peligroso dado que este comportamiento aumenta la probabilidad de colisión.

En otra pregunta, el 61\% de los conductores (3 de 5) respondió que la forma de manejar de los demás le hace perder la paciencia, como por ejemplo ir muy rápido o muy lento, cambiarse de carril bruscamente, no poner luces de 
estacionamiento o de dirección, etc., lo cual también es peligroso dado que estas acciones desvían la atención del conductor, aumentando la posibilidad de accidente. Por otro lado, este resultado contrasta con los resultados de otra pregunta, en la cual el $48 \%$ de los conductores (1 de 2) opinaron que con el poco tiempo que se tiene y el estado del tráfico, es justificable hacer maniobras arriesgadas al conducir. En otras palabras, algunos conductores justifican su comportamiento arriesgado, sin importar que ello pueda hacer perder la paciencia a otros conductores.

Los resultados de las dos preguntas anteriores pueden resumirse en que "yo lo estoy haciendo mejor que el resto". Eso también se puede ver en las respuestas de los conductores cuando se les preguntó acerca de qué tan buen conductor se considera, en las cuales, sólo el 14\% se considera como peor que la mayoría de conductores, mientras que el 58\% de los conductores se considera como un conductor promedio y el $28 \%$ se considera mejor que el promedio. Posiblemente también se deba a que 7 de cada 10 personas se considera prudente en temas relacionados a seguridad vial.

\section{Emociones de los encuestados}

La emoción es la alteración del ánimo intensa y pasajera, agradable o penosa, que va acompañada de cierta conmoción del cuerpo de la persona (Real Academia Española, 2014). Se evaluaron las emociones de los encuestados en tres escenarios: como peatón, conductor y acompañante, tal como se ve en las Figuras 1 al 3

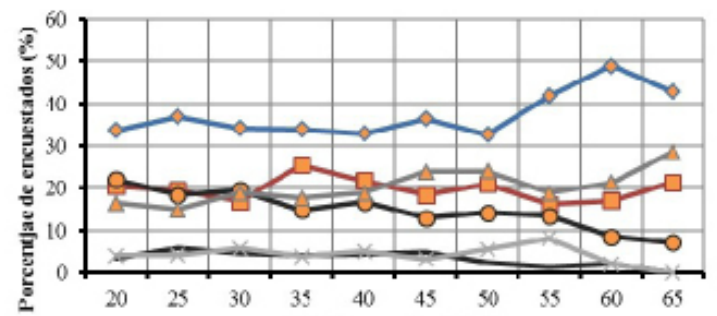

Figura 1. Emociones de los peàtones en función de la edad del encuestado

En la Figura 1 se puede ver que la emoción que prevalece en los peatones es el miedo, lo que es entendible considerando que son los usuarios más vulnerables del entorno vial.

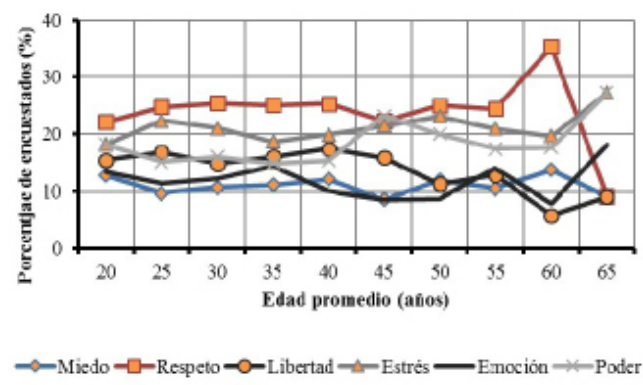

Figura 2. Emociones de los conductores en función de la edad del encuestado 
Por otro lado, la emoción que prevalece en los conductores es el respeto, seguido del estrés (ver Figura 2). Con la primera se confirma la premisa de que el conducir un vehículo está relacionado con el estatus social; y, con la segunda, se reafirma que la mayoría de conductores no disfrutan de la conducción, mostrado previamente

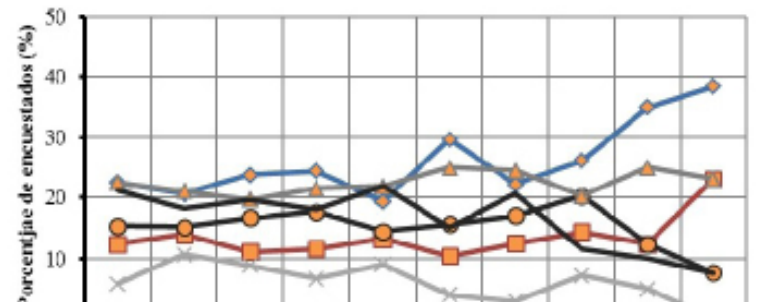

Figura 3. Emociones de los acompañantes o copilotos en función de la edad del encuestado

En lo que respecta a los acompañantes o copilotos (ver Figura 3) el miedo y el estrés son las emociones más mencionadas por los acompañantes o copilotos. Nótese que la proporción del miedo de los peatones es mayor a la proporción del miedo de los acompañantes o copilotos, debido a que percepción del encuestado, pues en caso de un accidente de tránsito, estar como peatón es menos "seguro" que estar dentro de un vehículo debido a la protección física que provee la carrocería del vehículo.

Asimismo, en las Figuras 1-3 también se puede ver que, en general, las emociones permanecen aproximadamente constantes hasta la edad promedio de 50 años, y a partir de ahí, las curvas de las emociones son más erráticas. De la misma forma, alrededor de esta edad, en la Figura1 y Figura 3 la proporción de personas que experimentan miedo cuando son peatones o copilotos aumenta significativamente, posiblemente se deba a que son uno de los grupos más vulnerables.

\section{Accidentes de tránsito}

En el 2016, se produjeron en Ecuador 30.269 accidentes de tránsito (ANT, 2016b) lo que llevó a 1967 fallecimientos reportados (ANT, 2016a). Estas estadísticas son de conocimiento público y pueden afectar la percepción del riesgo de la población. La percepción del riesgo es el producto de la evaluación de información interna y externa de la persona, con el fin de emitir un juicio en cuanto al peligro potencial que presenta una determinada situación vial (Martín \& Pingarrón, 2016). En lo que respecta a la sensación de riesgo de los encuestados, en promedio el 59\% respondió que es nada probable o poco probable que sufran un accidente de tránsito de cualquier tipo a lo largo del próximo año, el 25\% dijo que es medianamente probable y el $20 \%$ que es algo probable o muy probable. Asimismo, en promedio el 58\% de los encuestados respondió que es nada probable o poco probable que sufran alguna lesión o daño físico por un accidente de tránsito de cualquier tipo a lo largo del próximo año, el $23 \%$ dijo que es medianamente probable y el $18 \%$ 
que es algo probable o muy probable. Esto se conoce como optimismo irrealista, en donde las personas piensan que la posibilidad de tener un accidente de tránsito son significativamente menores que las posibilidades de sus semejantes (Martín \& Pingarrón, 2016).

La mayoría de personas sabe o piensa que los que consumen alcohol y los motociclistas son los que tienen una mayor cantidad de accidentes, mientras que personas como ellas son las que tiene menos probabilidad, tal como se muestra en la Figura 4. Con esto se confirma el optimismo irrealista de los encuestados. Asimismo, en la línea superior de la Figura 4, nótese que los encuestados más jóvenes piensan que los conductores que circulan bajo los efectos del alcohol son menos frecuentes que lo que opinan los encuestados mayores. Esta percepción podría llevar a un aumento del consumo de bebidas alcohólicas en jóvenes, dado que para ellos los accidentes de tránsito por consumo de alcohol son más bajos. Lo cual se evidencia en las estadísticas nacionales del 2014, en donde las edades de mayor consumo a nivel nacional fueron entre 19 y 24 años (INEC, 2014).

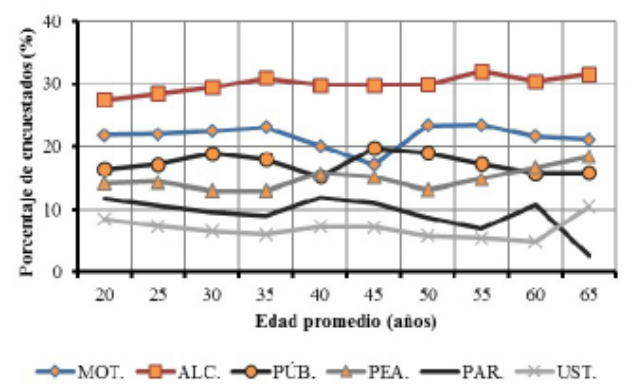

Figura4. Personas que tienen más accidentes de tránsito según la edad de los encuestados. MOT.: los motociclistas, ALC.: los que conducen bajo los efectos del alcohol, PÚB.: los conductores de transporte público,

Según la Figura 4, en promedio el 7\% cree que ellos son los que menos accidentes tienen, sin embargo el $40 \%$ admitió que tuvo 1 o más accidentes de tránsito en su vida. De ese $40 \%$, cada grupo de edad tuvo en promedio entre 1 y 2,40 accidentes de tránsito. Los que reportaron algún accidente de tránsito culparon a otra persona del accidente, es así que en promedio el 66\% culpó a otra persona del accidente, es decir 3 de cada 5 personas. De estos accidentes, el promedio el $46 \%$ piensa que fue accidental y el 54\% consideró que fue un error humano y que pudo evitarse.

Por otro lado, cuando se les preguntó acerca de los comportamientos, sólo el $5 \%$ dijo que la última vez que tuvo un comportamiento descuidado que hubiera podido ponerlo en peligro a ellos o a otras personas en la vía pública, fue hace una semana o menos; el 24\% entre 1 semana y 6 meses, el 21\% más de 6 meses, el 33\% no recuerda y el 16\% no cree que tenga comportamientos descuidados.

Asimismo, similares valores se obtuvieron cuando se les preguntó acerca de la última vez que tuvo un comportamiento peligroso que hubiera podido ponerlo en peligro a ellos o a otras personas en la vía pública, sólo el 5\% dijo que fue hace una semana o menos; el 21\% entre 1 semana y 6 meses, el $20 \%$ 
más de 6 meses, el 32\% no recuerda y el 23\% no cree que tenga comportamientos descuidados. Finalmente, cuando se les preguntó por cuándo fue la última vez que los encuestados hicieron algo no permitido, aunque haya sido inofensivo, la mayoría respondieron que no recuerdan.

\section{Leyes de tránsito}

En cuanto a las leyes de tránsito, el 96\% está de acuerdo que el principal requisito para ser un buen conductor o peatón es acatar la norma, ser precavido o estar a la defensiva, mientras que sólo el $4 \%$ respondieron que deben tomar ventaja de las oportunidades del tránsito para ahorrar tiempo o saber sortear toda situación de cualquier manera.

Por otra parte, el 14\% de los encuestados está de acuerdo con pocas normas y leyes de tránsito actual, el 54\% con algunas de ellas y el 32\% con la mayoría. El no estar de acuerdo con las normas y leyes puede llevar a su desobediencia, tal vez es por ello que en el 2016 se registró un 12,39\% de los accidentes de tránsito debido al irrespeto las leyes reglamentarias de tránsito (ANT, 2016b). Es que se debiera trabajar más en las leyes de tránsito dado que la mitad de las personas encuestadas (53\%) creen que la normativa de tránsito del Ecuador está muy atrasada y no responde a la realizada de la movilidad de nuestras ciudades.

En lo que respecta a cuán justas creen que son las leyes de tránsito actuales, el 21\% considera que las leyes son demasiado severas, el 21\% considera que las leyes son poco severas, el $25 \%$ considera que las leyes son apenas justas y el $26 \%$ considera que las leyes son muy justas. Por otro lado, más del 69\% dijo que no hay justificación para desobedecer las normas y leyes de tránsito; sin embargo, existieron algunas justificaciones para desobedecerlas, tal como se muestra en la Figura 5.

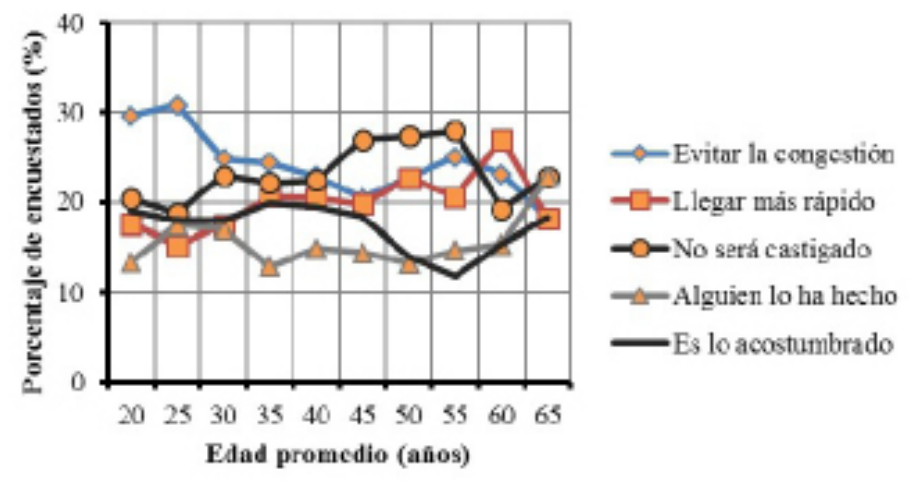

Figura 5. Justificaciones para desobedecer la ley según la edad de los encuestados

En la Figura 5 se puede observar, que cuando se trata de evitar la congestión, la tendencia de las respuestas muestra una disminución en porcentaje, conforme aumenta la edad del encuestado; es decir, los conductores más jóvenes justifican el quebrantar la ley con el fin de evitar la congestión más que los conductores mayores. Asimismo, en esta figura, la mayor proporción que justifica infringir la ley cuando se debe llegar más rápido fue para los encuestados con 60 años en promedio. Cuando se sabe que no será castigado, 
un mayor número de personas entre 45 y 55 respondieron afirmativamente. Y finalmente, cuando alguien lo ha hecho y es lo acostumbrado, fue la justificación que menos eligieron para la mayoría de edades consideradas.

\section{Consumo de alcohol}

Con respecto al consumo de alcohol durante la conducción, uno de cada tres personas encuestadas (32\%) piensa que un conductor sabe cuál es su límite y no debiera haber sanciones y prohibiciones tan extremas en el tema. En este mismo tema, uno de cada ocho personas encuestadas (12\%) piensa que no están bien las prohibiciones y sanciones que están en la ley de tránsito. Además, uno de cada cinco (20\%) no está de acuerdo con la cantidad de licor que se establece en la ley de tránsito. Todas estas respuestas muestran que parte de la población está en desacuerdo con la ley que incluye en consumo de bebidas alcohólicas, lo que puede conducir a la desobediencia, sin embargo, sólo el 6,92\% de las posibles causas de accidentes de tránsito en el 2016 a nivel nacional fue porque los conductores transitaban bajo la influencia del alcohol, drogas o medicamentos (ANT, 2016b). Cabe señalar que este porcentaje sólo se incluye los accidentes registrados por los agentes de tránsito, y además, pueden existir conductores que han circulado bajo los efectos del alcohol cuyo comportamiento no desembocó en accidente de tránsito.

\section{Límites de velocidad}

En el 2016, el 12,41\% (ANT, 2016b) de los accidentes de tránsito se produjeron por conducir a exceso de velocidad. Uno de cada cuatro encuestados (27\%) cree que los autos están diseñados para viajar a altas velocidades y no debería haber límites tan bajos y sanciones tan extremas. Asimismo, el 41\% ( 2 de 5 personas) está de acuerdo que están bien los límites de velocidad y las sanciones son pertinentes. Lo cual es coherente con que tres de cada cuatro personas (72\%) están de acuerdo con el límite de velocidad en las calles de 50 $\mathrm{km} / \mathrm{h}$ y tres de cada cinco personas (58\%) opinan que el límite de velocidad de $100 \mathrm{~km} / \mathrm{h}$ en carreteras es el adecuado. No obstante, existe un grupo de la población que no está de acuerdo con las regulaciones con respecto a los límites de velocidad, cuyo resultado puede ser la desobediencia, algo similar al consumo de bebidas alcohólicas o al respeto de leyes de tránsito.

\section{CONCLUSIONES}

Los resultados de los conductores que fueron más preocupantes estuvieron relacionados con: a) el estrés causado por la conducción, b) la justificación de sus maniobras arriesgadas, y c) el desacuerdo con las leyes de tránsito; mientras que los resultados de los peatones que fueron más preocupantes estuvieron relacionados con: a) el miedo que sienten cuando caminan por una calle, y c) el desacuerdo con las leyes de tránsito. Asimismo, se encontraron casos del optimismo realista en conductores y peatones, similar al encontrado en varios estudios preliminares. Estas percepciones, de los conductores y de los peatones, pueden afectar la frecuencia y gravedad de los accidentes de tránsito. Con respecto a las leyes de tránsito, es posible que muchas de las opiniones de los encuestados se deba a la falta de conocimiento, por ejemplo, 
alguien podría opinar que está de acuerdo con que se aumente la velocidad límite legal, a pesar de no conocer el castigo de circular a altas velocidades; o estar en desacuerdo con la reducción del límite de alcohol en la sangre cuando no se conocen los efectos del alcohol en la conducción. Ante ello, sería muy útil, que se establezcan campañas para explicar a la población la base teórica, con la cual se desarrollaron las leyes. En base a los análisis de los resultados obtenidos, se detectaron dos grupos de mayor riesgo: menores a 30 años y los mayores a 55 años, en los cuales debiera enfocarse las campañas de seguridad vial.

Finalmente se sugiere analizar la percepción de seguridad vial en puntos específicos de las calles, tales como intersecciones semaforizadas, ingreso a escuelas, salida de salas de cine, etc., dado que en esos lugares podrían afectar la percepción de la seguridad vial de la ciudadanía y cuyos resultados podría diferir de este estudio.

\section{AGRADECIMIENTO}

Los autores agradecen a la Secretaría Nacional de Educación Superior, Ciencia, Tecnología e Innovación (SENESCYT) de la República del Ecuador y a la Universidad Técnica Particular de Loja por la ayuda otorgada para el desarrollo de esta investigación.

\section{REFERENCIAS BIBLIOGRÁFICAS}

ANT. (2016a). Informe del número de fallecidos del 2016. Recuperado de http://www.ant.gob.ec/index.php/descargable/file/3906-fallecidos-diciembre-2016

ANT. (2016b). Informe del número de siniestros del 2016. Recuperado de http://www.ant.gob.ec/index.php/descargable/file/3905-siniestros-diciembre-2016

Código Orgánico Integral Penal (2014). Ministerio de Justicia, Derechos Humanos y Cultos. Recuperado de http://www.justicia.gob.ec/wp-content/ uploads/2014/05/código_orgánico_integral_penal_-_coip_ed._sdn-mjdhc. pdf

FAO. (2015). Densidad de población en América del Sur, Centroamérica y el Caribe. Recuperado de http://www.fao.org/nr/water/aquastat/countries regions/americas/figure02_esp.pdf

INEC. (2010). Resultados del censo 2010 de la población y vivienda del Ecuador. Fascículo provincial Loja. Recuperado de http://www.ecuadorencifras.gob.ec//wp-content/descargas/Manu-lateral/Resultados-provinciales/loja.pdf

INEC. (2014, March). Postdata "La realidad tras las cifras." Recuperado de http://www.ecuadorencifras.gob.ec/documentos/web-inec/Revistas/Postdata/postdata01/\#/1/

IPSOS. (2010). Primer estudio de comportamiento y seguridad vial ficha téc- 
nica. Recuperado de http://autozuniga.com/wp-content/uploads/2015/07/ Estudio-de-comportamiento-y-seguridad-vial.pdf

Ley N 744 (2016). Quito: Registro oficial. Órgano de Gobierno del Ecuador. Recuperado de https://spryn.finanzas.gob.ec/esipren-web/archivos_html/ file/RO-Ley-Equilibrio-Finanzas-Publicas.pdf

Martín, B., \& Pingarrón, A. (2016). La formación de los conductores como factor de Seguridad Vial. En V Congreso Ibero-americano de Seguridad Vial. Santiago de Chile. Recuperado de http://www.institutoivia.com/cisev-ponencias/formacion_seguridad_ev/Escurin_Pingarron.pdf

OMS. (2015). Global status report on road safety 2015. Recuperado de http:// www.who.int/violence_injury_prevention/road_safety_status/2015/en/

ONU. (2011). Plan mundial para el decenio de acción para la seguridad vial 2011-2020. Recuperado de http://www.who.int/roadsafety/decade_of_action/plan/spanish.pdf

Real Academia Española. (2014). Diccionario de la lengua española. Recuperado de http://dle.rae.es/

Wegman, F. (2017). The future of road safety: A worldwide perspective. IATSS Research, 40(2), 66-71. http://doi.org/10.1016/j.iatssr.2016.05.003 